\title{
CONFORMAL GEOMETRY OF THE (DISCRETE) SCHWARZIAN DAVEY-STEWARTSON II HIERARCHY
}

\author{
B. G. KONOPELCHENKO \\ Dipartimento di Fisica, Università di Lecce and Sezione INFN, 73100 Lecce, Italy \\ and W. K. SCHIEF \\ School of Mathematics, The University of New South Wales, Sydney, NSW 2052, Australia
}

(Received 30 November, 2003; accepted 1 July, 2004)

\begin{abstract}
The conformal geometry of the Schwarzian Davey-Stewartson II hierarchy and its discrete analogue is investigated. Connections with discrete and continuous isothermic surfaces and generalised Clifford configurations are recorded. An interpretation of the Schwarzian Davey-Stewartson II flows as integrable deformations of conformally immersed surfaces is given.
\end{abstract}

2000 Mathematics Subject Classification. 53A30, 35Q58.

1. Introduction. Due to the (re-)discovery of a variety of important connections between the differential geometry of surfaces and integrable systems, (classical and modern) differential geometry has been widely recognised as an integral part of soliton theory (see, e.g., [1]-[3]). However, the fundamental nature of geometry in the context of integrable systems is a subject of ongoing research and recent investigations have uncovered unexpected geometric links. For instance, it has been established that Hirota's master equation [4] in its Schwarzian form and the associated scalar Schwarzian Kadomtsev-Petviashvili (SKP) hierarchy are encapsulated in Menelaus' classical theorem of plane geometry [5]-[7].

In the present paper, we embark on a study of the geometry of the Schwarzian Davey-Stewartson II hierarchy and its discrete analogue, the quaternionic discrete SKP (qdSKP) equation. We demonstrate that the qdSKP equation and various associated continuum limits are canonical objects of conformal (Möbius) geometry in $\mathbb{R}^{4}$. In particular, we establish important connections with both discrete and continuous isothermic surfaces and generalised Clifford point-circle configurations. We also show that the Schwarzian Davey-Stewartson II hierarchy explicitly defines integrable deformations of conformal immersions in $\mathbb{R}^{4}$.

2. The multicomponent discrete Schwarzian KP equation. The multicomponent KP hierarchy houses a variety of important soliton equations such as the DaveyStewartson and $N$-wave equations and their associated hierarchies [8]. The Schwarzian KP (SKP) hierarchy consisting of the singularity manifold equations for the multicomponent KP hierarchy has been shown to admit an elegant compact formulation [9]. Indeed, it has been established that if an $N \times N$ matrix

$$
\Phi(t), \quad t=\left(t_{1}, t_{2}, t_{3}, \ldots\right)
$$


depending on an infinite number of 'times' $t_{n}$ constitutes a solution of the SKP hierarchy then the six solutions

$$
\Phi_{i}=T_{i} \Phi, \quad \Phi_{i k}=T_{i} T_{k} \Phi, \quad i, k=1,2,3, \quad i \neq k,
$$

where the 'shift' operators $T_{i}$ are defined by

$$
T_{i} \Phi(t)=\Phi\left(t+\left[a_{i}\right]\right)=\Phi\left(t_{1}+a_{i}, t_{2}+\frac{a_{i}^{2}}{2}, t_{3}+\frac{a_{i}^{3}}{3}, \ldots\right)
$$

and $a_{i}=$ const, obey the algebraic 6-point relation

$$
\mathrm{M}\left(\Phi_{1}, \Phi_{12}, \Phi_{2}, \Phi_{23}, \Phi_{3}, \Phi_{31}\right)=-\mathbb{1} .
$$

Here, the multi-ratio $\mathrm{M}$ of six matrices $P^{1}, \ldots, P^{6}$ is defined by

$$
\begin{aligned}
& \mathrm{M}\left(P^{1}, P^{2}, P^{3}, P^{4}, P^{5}, P^{6}\right) \\
& \quad=\left(P^{1}-P^{2}\right)\left(P^{2}-P^{3}\right)^{-1}\left(P^{3}-P^{4}\right)\left(P^{4}-P^{5}\right)^{-1}\left(P^{5}-P^{6}\right)\left(P^{6}-P^{1}\right)^{-1} .
\end{aligned}
$$

The entire SKP hierarchy may then be retrieved from (4) by considering a canonical limit in which $a_{i} \rightarrow 0$ [9]-[11].

As indicated above, the multi-ratio relation (4) represents an algebraic superposition formula for six solutions of the multicomponent SKP hierarchy. As in the scalar case $[\mathbf{5}, \mathbf{1 0}]$, it also constitutes an algebraic relation ('permutability theorem') for six solutions generated by a variant of the classical Darboux transformation $[\mathbf{1 2}, 13]$. Iterative application of the Darboux transformation then produces lattices of solutions of the multicomponent SKP hierarchy. In particular, the multi-ratio relation (4) may be interpreted as an equation defined on a $\mathbb{Z}^{3}$ lattice. Thus, in the following, we regard (4) as a discrete equation for a matrix-valued function

$$
\Phi: \mathbb{Z}^{3} \rightarrow \mathbb{C}^{N, N}, \quad\left(n_{1}, n_{2}, n_{3}\right) \mapsto \Phi\left(n_{1}, n_{2}, n_{3}\right),
$$

where the indices on $\Phi$ denote translations on the lattice, that is, for instance,

$$
\Phi=\Phi\left(n_{1}, n_{2}, n_{3}\right), \quad \Phi_{1}=\Phi\left(n_{1}+1, n_{2}, n_{3}\right), \quad \Phi_{23}=\Phi\left(n_{1}, n_{2}+1, n_{3}+1\right) .
$$

The discrete equation (4) has come to be known as the multicomponent discrete SKP (dSKP) equation since it encodes the complete multicomponent SKP hierarchy. Indeed, the genesis of the multicomponent dSKP equation implies that any member of the multicomponent SKP hierarchy may be obtained from (4) by applying an appropriate continuum limit. The integrable nature of the multicomponent dSKP equation is inherited from both the original derivation (1)-(4) and the construction via Darboux transformations. The following analysis is concerned with the conformal geometry of the quaternionic dSKP equation and its various continuum limits.

3. The quaternionic dSKP equation. An important property of the multicomponent dSKP equation is its invariance under fractional linear transformations of the form

$$
\Phi \rightarrow \Phi^{\prime}=(A \Phi+B)(C \Phi+D)^{-1},
$$


where $A, B, C$ and $D$ are arbitrary constant matrices. It is therefore natural to investigate whether contact may be made with conformal (differential) geometry. Thus, in the remainder of the paper, we assume that the matrix $\Phi$ takes values in the space of quaternions $\mathbb{H}$. The latter is identified with a four-dimensional Euclidean space $\mathbb{R}^{4}$ via

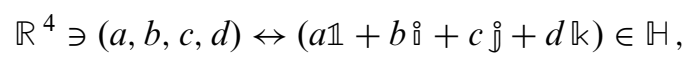

where the matrices $\mathbb{1}, \stackrel{\circ}{,}, \stackrel{\mathbb{D}}{\mathbb{k}} \mathbb{k}$ are defined by

$$
\mathbb{1}=\left(\begin{array}{ll}
1 & 0 \\
0 & 1
\end{array}\right), \quad \AA=\left(\begin{array}{cc}
0 & -\mathrm{i} \\
-\mathrm{i} & 0
\end{array}\right), \quad \AA=\left(\begin{array}{cc}
0 & -1 \\
1 & 0
\end{array}\right), \quad \mathbb{k}=\left(\begin{array}{cc}
-\mathrm{i} & 0 \\
0 & \mathrm{i}
\end{array}\right) .
$$

In particular, this isomorphism gives rise to the identities

$$
\boldsymbol{X}^{2}=\operatorname{det} X, \quad X X^{\dagger}=(\operatorname{det} X) \mathbb{1}, \quad \boldsymbol{X} \cdot \boldsymbol{Y}=\frac{1}{2} \operatorname{tr}\left(X Y^{\dagger}\right)
$$

for any quaternions $X, Y \in \mathbb{H}$ and their vectorial analogues $X, Y \in \mathbb{R}^{4}$. Accordingly, in the case $\Phi \in \mathbb{H}$, we may refer to (4) as the quaternionic dSKP (qdSKP) equation. The invariance (8) encodes the group of orientation-preserving conformal transformations in $\mathbb{R}^{4}$ provided that $A, B, C$ and $D$ are quaternions.

3.1. The 'geometric' continuum limit. As alluded to in the preceding section, the complete quaternionic SKP hierarchy may be retrieved from the qdSKP equation via appropriate sophisticated limits. We embark on a study of the geometric implications of this fact in the next section. Here, by contrast, we focus on the natural 'geometric' limit in which the differences $\Delta_{i} \Phi=\Phi_{i}-\Phi$ are regarded as approximations of derivatives, that is

$$
\Phi_{i}=\Phi+\epsilon \Phi_{x_{i}}+O\left(\epsilon^{2}\right), \quad i=1,2,3
$$

where $\epsilon$ is a lattice parameter and $\Phi_{x_{i}}=\partial \Phi / \partial x_{i}$. In the limit $\epsilon \rightarrow 0$ and $\left(x_{1}, x_{2}, x_{3}\right)=$ $(x, y, z)$, the qdSKP equation reduces to

$$
\Phi_{y} \Phi_{x}^{-1} \Phi_{z} \Phi_{y}^{-1} \Phi_{x} \Phi_{z}^{-1}=\mathbb{1}
$$

The latter becomes an identity if the symmetry

$$
\Phi_{y}+\Phi_{z}=0
$$

is imposed. The scalar analogue of this constraint has been shown to lead to conformal maps or (anti-)analytic functions on the complex plane [5]. As in the scalar case, in order to obtain a non-trivial continuum limit from the qdSKP equation subject to the constraint (14), it is required to take into account second-order terms in the Taylor expansion (12) and consider the terms in the expansion of the qdSKP equation which 
are linear in $\epsilon$, that is

$$
\begin{aligned}
{\left[\Phi_{x y}\right.} & \left.+\frac{1}{2} \Phi_{y y}-\Phi_{y} \Phi_{x}^{-1}\left(\Phi_{x y}+\frac{1}{2} \Phi_{x x}\right)\right] \Phi_{x}^{-1} \Phi_{z} \Phi_{y}^{-1} \Phi_{x} \Phi_{z}^{-1} \\
& +\Phi_{y} \Phi_{x}^{-1}\left[\Phi_{y z}+\frac{1}{2} \Phi_{z z}-\Phi_{z} \Phi_{y}^{-1}\left(\Phi_{y z}+\frac{1}{2} \Phi_{y y}\right)\right] \Phi_{y}^{-1} \Phi_{x} \Phi_{z}^{-1} \\
& +\Phi_{y} \Phi_{x}^{-1} \Phi_{z} \Phi_{y}^{-1}\left[\Phi_{x z}+\frac{1}{2} \Phi_{x x}-\Phi_{x} \Phi_{z}^{-1}\left(\Phi_{x z}+\frac{1}{2} \Phi_{z z}\right)\right] \Phi_{z}^{-1}=0
\end{aligned}
$$

Simplification by means of (14) then yields

$$
\Phi_{x y}=\frac{1}{2} \Phi_{x} \Phi_{y}^{-1} \Phi_{y y}+\frac{1}{2} \Phi_{y y} \Phi_{y}^{-1} \Phi_{x} .
$$

By virtue of the isomorphism $\mathbb{R}^{4} \cong \mathbb{H}$, any solution of the quaternionic equation (16) gives rise to a surface immersed in $\mathbb{R}^{4}$ and parametrised by the coordinates $x$ and $y$. Its position vector $\boldsymbol{r}=(r, r) \in \mathbb{R}^{4}$ is obtained from the decomposition

$$
\Phi=r \mathbb{1}+\mathrm{r} \cdot \mathrm{e}
$$

where the 'vector' $\mathrm{e}$ is defined by $\mathrm{e}=(\stackrel{\circ}{0}, \mathfrak{\rrbracket}, \mathbb{k})$. In terms of $\boldsymbol{r}$, equation (16) is readily seen to translate into

$$
\boldsymbol{r}_{x y}=\frac{\boldsymbol{r}_{y} \cdot \boldsymbol{r}_{y y}}{\boldsymbol{r}_{y}^{2}} \boldsymbol{r}_{x}-\frac{\boldsymbol{r}_{x} \cdot \boldsymbol{r}_{y y}}{\boldsymbol{r}_{y}^{2}} \boldsymbol{r}_{y}+\frac{\boldsymbol{r}_{x} \cdot \boldsymbol{r}_{y}}{\boldsymbol{r}_{y}^{2}} \boldsymbol{r}_{y y} .
$$

Thus, in the natural geometric continuum limit, the qdSKP equation subject to the symmetry constraint (14) governs surfaces the position vector of which obeys the second-order equation (18).

By construction, the surfaces defined above belong to conformal differential geometry (see, e.g., [14]) due to the invariance of (18) under the group of conformal (Möbius) transformations as induced by (8). These are integrable in the sense that they are given in terms of solutions of the soliton equation (16). In order to proceed, it is now observed that (16) implies that

$$
Q_{y}=\frac{1}{2}\left[\Phi_{y y} \Phi_{y}^{-1}, Q\right]
$$

where the quaternion $Q$ is defined by

$$
Q=\Phi_{x} \Phi_{y}^{-1} \Phi_{x} \Phi_{y}^{-1}
$$

It may therefore be admissible to impose the constraint

$$
\left(\Phi_{x} \Phi_{y}^{-1}\right)^{2}=-\mathbb{1}
$$

since, in this case, relation (19) is identically satisfied. In the generic case, that is $\Phi_{x} \nsucc \Phi_{y}$, the constraint (21) is equivalent to the pair

$$
\operatorname{tr}\left(\Phi_{x} \Phi_{y}^{-1}\right)=0, \quad \operatorname{det}\left(\Phi_{x} \Phi_{y}^{-1}\right)=1
$$


which, in terms of the position vector $\boldsymbol{r}$, becomes

$$
\boldsymbol{r}_{x}^{2}=\boldsymbol{r}_{y}^{2}, \quad \boldsymbol{r}_{x} \cdot \boldsymbol{r}_{y}=0
$$

The latter conditions may be used to cast (18) into the form

$$
\boldsymbol{r}_{x y}=\frac{\boldsymbol{r}_{x} \cdot \boldsymbol{r}_{x y}}{\boldsymbol{r}_{x}^{2}} \boldsymbol{r}_{x}+\frac{\boldsymbol{r}_{y} \cdot \boldsymbol{r}_{x y}}{\boldsymbol{r}_{y}^{2}} \boldsymbol{r}_{y}
$$

or, equivalently,

$$
\boldsymbol{r}_{x y}=a \boldsymbol{r}_{x}+b \boldsymbol{r}_{y}
$$

where the real coefficients $a$ and $b$ are determined by the constraints (23). Thus, it has been established that the surfaces defined by the system (23), (25) constitute a subclass of the surfaces associated with the continuum limit of the qdSKP equation considered here.

The geometry of the reduction (23), (25) is now readily revealed. Firstly, the constraints (23) are equivalent to demanding that the first fundamental form of a surface be conformally flat, that is

$$
d \boldsymbol{r}^{2}=\Omega\left(d x^{2}+d y^{2}\right), \quad \Omega=\boldsymbol{r}_{x}^{2}=\boldsymbol{r}_{y}^{2} .
$$

The coordinates $x$ and $y$ are therefore conformal coordinates [15]. Secondly, the hyperbolic equation (25) expresses the fact that the vector $\boldsymbol{r}_{x y}$ is tangential to the surface so that the coordinates $x$ and $y$ are conjugate [15]. A surface which may be parametrised simultaneously in terms of conformal and conjugate coordinates is termed an isothermic surface. ${ }^{\dagger}$ Hence, we conclude that the continuum limit (16) of the qdSKP equation subject to the constraint (21) is associated with isothermic surfaces. The latter are classical and have been investigated extensively with respect to both geometry and integrability (see, e.g., $[2,3]$ and references therein). The connection with isothermic surfaces therefore provides a first indication of the fundamental nature of the qdSKP equation.

3.2. The conformal geometry of the qdSKP equation. Discrete isothermic surfaces. The qdSKP equation has recently been given a geometric interpretation [16] in terms of a novel generalisation of Clifford's classical $\mathcal{C}_{4}$ point-circle configuration. A $\mathcal{C}_{4}$ Clifford configuration is constructed in the following manner $[\mathbf{1 7}, \mathbf{1 8}]$ : Consider a point $P^{0}$ on a plane and four generic coplanar circles $S^{1}, S^{2}, S^{3}, S^{4}$ passing through $P^{0}$. The additional six points of intersection are labelled by $P^{12}, P^{13}, P^{14}, P^{23}, P^{24}, P^{34}$, where the indices on $P^{i k}$ correspond to those of the intersecting circles $S^{i}$ and $S^{k}$ (cf. Figure 1). Any three circles $S^{i}, S^{k}, S^{l}$ intersect in three points and therefore define a circle $S^{i k l}$ passing through the points of intersection $P^{i k}, P^{i l}, P^{k l}$. Clifford's circle theorem then states that, remarkably, the four circles $S^{123}, S^{124}, S^{134}, S^{234}$ meet at a point $P^{1234}$. It is noted in passing that Clifford configurations $\left(\mathcal{C}_{n}\right)$ exist for any number of initial circles $S^{1}, \ldots, S^{n}$ passing through a point $P^{0}$.

${ }^{\dagger}$ Accordingly, a surface is isothermic if and only if its curvature coordinates are conformal modulo a suitable reparametrisation $x \rightarrow f(x), y \rightarrow g(y)[\mathbf{1 5}]$. 


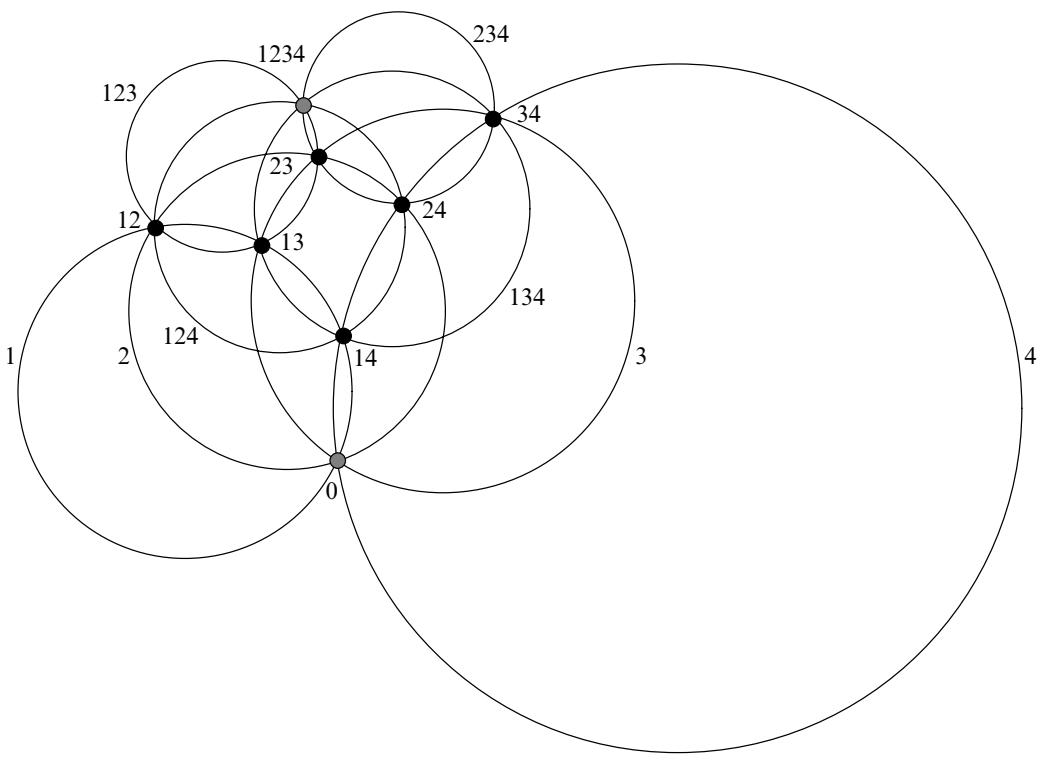

Figure 1. A $\mathcal{C}_{4}$ Clifford configuration

In [5], it has been shown that the six points $P^{i k}$ of a $\mathcal{C}_{4}$ Clifford configuration are algebraically related by the multi-ratio relation

$$
\mathrm{M}\left(P^{14}, P^{12}, P^{24}, P^{23}, P^{34}, P^{13}\right)=-1
$$

if the plane is identified with the complex plane so that the points $P^{i k}$ are regarded as complex numbers. The above multi-ratio relation encodes nothing but Menelaus' classical theorem of plane geometry if the point $P^{0}$ is mapped to infinity by a conformal transformation [19]. Here, it is important to note that if we set aside the points $P^{0}$ and $P^{1234}$ then a $\mathcal{C}_{4}$ Clifford configuration exhibits the combinatorics of an octahedron if the six points $P^{i k}$ and eight circles $S^{i}, S^{i k l}$ are identified with the vertices and faces of an octahedron respectively. In particular, the multi-ratio relation (27) admits the full symmetry group of an octahedron acting on the entries $P^{i k}$. Moreover, Ziegenbein [18] has proven that all circles and points of a $\mathcal{C}_{4}$ Clifford configuration appear on equal footing in the sense that the angles made by four oriented circles passing through a point are the same for all eight points. The converse of Ziegenbein's theorem is also valid.

It has been established in [5] that any 'generic' six points $P^{i k}$ on the complex plane belong to a $\mathcal{C}_{4}$ Clifford configuration if and only if the multi-ratio relation (27) is satisfied. It is therefore natural to inquire as to the geometric significance of the quaternionic multi-ratio condition

$$
\mathrm{M}\left(P^{14}, P^{12}, P^{24}, P^{23}, P^{34}, P^{13}\right)=-\mathbb{1},
$$

where the quaternions $P^{i k}$ are regarded as points in a four-dimensional Euclidean space. It is evident that for any 'generic' six points $P^{i k} \in \mathbb{R}^{4}$, the circles $S^{i}$ and $S^{i k l}$ may still be constructed but, in general, these do not intersect in any points $P^{0}$ and $P^{1234}$. However, if one imposes the Ziegenbein property then the six points $P^{i k}$ can no longer 
be arbitrary. In fact, it turns out [16] that the Ziegenbein property is equivalent to the quaternionic multi-ratio condition. ${ }^{\dagger}$ Thus, the latter condition gives rise to generalised $\mathcal{C}_{4}$ Clifford configurations if their definition is based on the Ziegenbein property.

The preceding discussion of Clifford configurations implies that the qdSKP equation

$$
\mathrm{M}\left(\Phi_{1}, \Phi_{12}, \Phi_{2}, \Phi_{23}, \Phi_{3}, \Phi_{31}\right)=-\mathbb{1} .
$$

enshrines 'collections' of generalised $\mathcal{C}_{4}$ Clifford configurations. This has been made precise in [16]. On the other hand, the connection with classical isothermic surfaces as established in Section 3.1 raises the question as to whether the standard integrable discretisation of isothermic surfaces [2] is related to generalised Clifford configurations. A quadrilateral lattice (discrete surface)

$$
\Phi: \mathbb{Z}^{2} \rightarrow \mathbb{R}^{4} \cong \mathbb{H}
$$

is termed isothermic if the quaternionic cross-ratio

$$
Q\left(\Phi, \Phi_{1}, \Phi_{12}, \Phi_{2}\right)=\left(\Phi-\Phi_{1}\right)\left(\Phi_{1}-\Phi_{12}\right)^{-1}\left(\Phi_{12}-\Phi_{2}\right)\left(\Phi_{2}-\Phi\right)^{-1}
$$

associated with any quadrilateral obeys

$$
Q\left(\Phi, \Phi_{1}, \Phi_{12}, \Phi_{2}\right)=-\mathbb{1}
$$

Since the above quaternionic cross-ratio condition is invariant under conformal transformations and any four points in $\mathbb{R}^{4}$ may be mapped to the plane by means of an appropriate conformal transformation, the quadrilaterals are inscribed in circles and their classical (scalar) cross-ratio is -1 [2]. This is the geometric content of the quaternionic cross-ratio condition (32). It is observed that the quaternionic cross-ratio condition constitutes a natural discretisation of the constraint (21). However, since the quadrilaterals are planar, it also discretises the hyperbolic equation (25) so that (32) may indeed be regarded as a discrete version of the conditions (23), (25) defining classical isothermic surfaces [2].

The canonical discrete analogue of the constraint (14) is given by the translational symmetry

$$
\Phi_{23}=\Phi
$$

The latter may be used to eliminate quantities which carry an index 3 from the qdSKP equation (29). On rearranging terms, one obtains

$$
Q\left(\Phi_{1}, \Phi_{12}, \Phi_{2}, \Phi\right)=T_{\overline{2}} Q\left(\Phi_{12}, \Phi_{1}, \Phi, \Phi_{2}\right),
$$

where $T_{\overline{2}} f\left(n_{2}\right)=f\left(n_{2}-1\right)$. If the quaternionic cross-ratio condition (32) holds then both cross-ratios in (34) are $-\mathbb{1}$ due to the symmetry group of the cross-ratio condition. Accordingly, the pair (32), (33) constitutes a reduction of the qdSKP equation. In geometric terms, this implies that any discrete isothermic surface extended to a threedimensional lattice via the translational symmetry (33) represents a collection of (degenerate) generalised $\mathcal{C}_{4}$ Clifford configurations as introduced in [16] and alluded to in the preceding.

\footnotetext{
${ }^{\dagger}$ Modulo an inversion with respect to a hypersphere.
} 
4. Deformation of conformal immersions induced by the Schwarzian DaveyStewartson II hierarchy. In order to investigate the conformal differential geometry of the continuous qSKP hierarchy, we now recall the connection between the qdSKP equation and the (adjoint) eigenfunctions associated with the quaternionic discrete KP equation, that is the discrete Davey-Stewartson II equation [9]-[11]. Thus, it is readily verified that the linear system

$$
\phi_{2}=\phi_{1}\left(\Delta_{1} \Phi\right)^{-1} \Delta_{2} \Phi, \quad \phi_{3}=\phi_{1}\left(\Delta_{1} \Phi\right)^{-1} \Delta_{3} \Phi
$$

for a quaternionic function $\phi$ is compatible modulo the qdSKP equation (29). Hence, one may introduce a quaternionic function of 'separation' $\psi$ according to

$$
\Delta_{i} \Phi=\psi \phi_{i}
$$

The compatibility conditions $\left[\Delta_{i}, \Delta_{k}\right] \Phi=0$ then yield

$$
\left(\phi_{i}-\phi_{k}\right) \phi_{i k}^{-1}=\psi^{-1}\left(\psi_{k}-\psi_{i}\right) \text {. }
$$

On the one hand, addition of the three relations (37) produces

$$
\left(\phi_{1}-\phi_{2}\right) \phi_{12}^{-1}+\left(\phi_{2}-\phi_{3}\right) \phi_{23}^{-1}+\left(\phi_{3}-\phi_{1}\right) \phi_{31}^{-1}=0
$$

which constitutes the eigenfunction equation for the quaternionic discrete KP equation. The latter represents nothing but a discrete Davey-Stewartson II equation so that (38) may be regarded as a discrete version of the modified Davey-Stewartson II equation which is known as the Ishimori equation [20]. On the other hand, elimination of the eigenfunction $\phi$ from (37) in a similar manner gives rise to the 'adjoint' eigenfunction equation

$$
\psi_{1}^{-1}\left(\psi_{31}-\psi_{12}\right)+\psi_{2}^{-1}\left(\psi_{12}-\psi_{23}\right)+\psi_{3}^{-1}\left(\psi_{23}-\psi_{31}\right)=0
$$

with $\psi$ being an adjoint eigenfunction of the discrete Davey-Stewartson II equation. It is emphasised that the qdSKP and (adjoint) quaternionic discrete KP eigenfunction equations are equivalent. For instance, if $\phi$ is an eigenfunction obeying (38) then the linear system (37) for $\psi$ is compatible and $\psi$ constitutes a solution of the adjoint eigenfunction equation (39). Moreover, by construction, the existence of a function $\Phi$ satisfying the defining relations (36) is guaranteed and $\Phi$ is indeed a solution of the qdSKP equation (29).

The continuum limit to the qSKP hierarchy requires the introduction of the gauge transformations

$$
\phi \rightarrow A_{1}^{n_{1}} A_{2}^{n_{2}} A_{3}^{n_{3}} \phi, \quad \psi \rightarrow \psi A_{3}^{-n_{3}} A_{2}^{-n_{2}} A_{1}^{-n_{1}},
$$

where the constant matrices $A_{i}$ constitute non-degenerate diagonal quaternions. In the limit in which the qdSKP equation reduces to the $n$ th-order qSKP equation, the system (36) becomes [9]-[11]

$$
\Phi_{x}=\psi A_{1} \phi, \quad \Phi_{y}=\psi A_{2} \phi, \quad \Phi_{t}=\mathcal{B}_{n}(\psi, \phi),
$$

where $\mathcal{B}_{n}$ is bilinear in $\psi, \phi$ and their derivatives. If we make the choice $A_{1}=\mathbb{1}$ and $A_{2}=\mathbb{k}$ and eliminate the eigenfunction $\phi$ from (41) then we obtain the pair

$$
\Phi_{y}=N \Phi_{x}, \quad \Phi_{t}=\mathcal{L}_{n}(\Phi),
$$


where

$$
N=\psi k \psi^{-1}
$$

and $\mathcal{L}_{n}(\Phi)=\mathcal{B}_{n}\left(\psi, \psi^{-1} \Phi_{x}\right)$. Accordingly, $\mathcal{L}_{n}$ constitutes a linear operator acting on $\Phi$ with coefficients depending on $\psi$ and its derivatives. It turns out that these coefficients may be written as differential expressions in $N$ and a scalar auxiliary function $\varphi$ [9]-[11]. The compatibility condition for the linear system (42) then gives rise to the $n$ th-order Ishimori equation. For instance, in the case $n=2$, the Lax pair (42) assumes the form

$$
\Phi_{y}=N \Phi_{x}, \quad \Phi_{t}=N\left(\Phi_{x y}-\varphi_{y} \Phi_{x}-\varphi_{x} \Phi_{y}\right)
$$

and its compatibility condition yields

$$
N_{t}=N N_{x y}+N_{y} N_{x}+\varphi_{x} N_{x}-\varphi_{y} N_{y}-\left(\varphi_{x x}+\varphi_{y y}\right) N .
$$

Now, the definition (43) of $N$ implies that

$$
\operatorname{tr} N=0, \quad N^{2}=-\mathbb{1}, \quad N=\mathrm{S} \cdot \mathrm{e}, \quad \mathrm{S}^{2}=1
$$

and hence decomposition of the matrix equation (45) produces

$$
\begin{gathered}
\mathbf{S}_{t}=\mathbf{S} \times \mathbf{S}_{x y}+\varphi_{x} \mathbf{S}_{x}-\varphi_{y} \mathbf{S}_{y} \\
\varphi_{x x}+\varphi_{y y}+\left(\mathbf{S}_{x} \times \mathbf{S}_{y}\right) \cdot \mathbf{S}=0 .
\end{gathered}
$$

The latter represents the Ishimori equation which was first set down in [20]. It is interesting to note that the 'topological charge'

$$
\mathrm{Q}=\frac{1}{4 \pi} \int\left(\mathrm{S}_{x} \times \mathrm{S}_{y}\right) \cdot \mathrm{S} d x d y
$$

is preserved by the Ishimori flow $(47)_{1}$. In fact, the topological charge may be shown to be invariant under all higher-order Ishimori flows. It is also remarked that the Ishimori hierarchy is amenable to the inverse spectral transform (IST) method [21, 22].

The geometry of the Schwarzian Davey-Stewartson (SDS) II hierarchy encoded in the Lax pair (42) for $n=2,3, \ldots$ is unveiled by focussing on the 'scattering problem' $(42)_{1}$ (cf. [23]). Thus, if, for any fixed $t$, we identify the quaternionic function $\Phi$ with the position vector $\boldsymbol{r}$ of a surface in $\mathbb{R}^{4}$ then the properties $(46)_{1,2}$ show that the metric of the surface is once again given by (26) with $x$ and $y$ being conformal coordinates. Any SDSII flow (42) $)_{2}$ therefore defines an integrable deformation of conformal immersions of surfaces in $\mathbb{R}^{4}$, where the independent variable $t$ is regarded as the deformation parameter. The associated Ishimori flows possess an infinite set of invariants, the simplest of which is given by the topological charge (48). Moreover, the invariance of the qdSKP equation under the Möbius transformation (8) guarantees that the SDSII deformations are covariant under conformal transformations in $\mathbb{R}^{4}$. For instance, the Lax pair (44) is form-invariant under

$$
\Phi \rightarrow \Phi^{-1}, \quad N \rightarrow \Phi^{-1} N \Phi, \quad \varphi \rightarrow \varphi-\ln \operatorname{det} \Phi
$$

corresponding to the composition of an inversion and a reflection in $\mathbb{R}^{4}$ given by

$$
\boldsymbol{r}=(r, \mathrm{r}) \rightarrow\left(\frac{r}{\boldsymbol{r}^{2}},-\frac{\mathrm{r}}{\boldsymbol{r}^{2}}\right), \quad \Omega \rightarrow \frac{\Omega}{\boldsymbol{r}^{4}}
$$


An important feature of the immersion $(42)_{1}$ and the deformations $(42)_{2}$ is that these are formulated explicitly in terms of geometric quantities, namely the position vector $\boldsymbol{r}$ (or $\Phi$ ) and the quantity $N$. The latter is known as the 'left normal' of the surface [23]. If $\operatorname{tr} \Phi=0$, that is if the surface is embedded in $\mathbb{R}^{3} \cong \operatorname{Im} H$, then the immersion formula $(42)_{1}$ decomposes into

$$
\mathrm{r}_{y}=\mathrm{S} \times \mathrm{r}_{x}, \quad \mathrm{~S} \cdot \mathrm{r}_{x}=0
$$

so that $\mathrm{S}$ constitutes the unit normal

$$
\mathrm{S}=\frac{\mathrm{r}_{x} \times \mathrm{r}_{y}}{\Omega}
$$

to the surface. However, in general, $N$ is not normal to the surface and the constraint $\operatorname{tr} \Phi=0$ is not preserved by the SDSII flows $(42)_{2}$. In this connection, it is interesting to investigate the stationary points of the SDSII deformations. For instance, if $\Phi_{t}=0$ then the flow $(44)_{2}$ reduces to

$$
\Phi_{x y}=\varphi_{y} \Phi_{x}+\varphi_{x} \Phi_{y}
$$

which is nothing but the hyperbolic equation (24) with

$$
\varphi=\frac{1}{2} \ln \Omega \text {. }
$$

Thus, the class of surfaces which is preserved by the deformation associated with the Schwarzian Davey-Stewartson II equation coincides with the class of isothermic surfaces in $\mathbb{R}^{4}$. The metric of any such surface is given by

$$
d \boldsymbol{r}^{2}=e^{2 \varphi}\left(d x^{2}+d y^{2}\right)
$$

which affords an immediate geometric interpretation of the auxiliary function $\varphi$. Moreover, the auxiliary equation $(47)_{2}$ constitutes the Gauß equation [15]

$$
\varphi_{x x}+\varphi_{y y}+\mathcal{K} e^{2 \varphi}=0,
$$

where the Gaußian curvature $\mathcal{K}$ of the surface is given by

$$
\mathcal{K}=\left(\mathrm{S}_{x} \times \mathrm{S}_{y}\right) \cdot \mathrm{S} e^{-2 \varphi} .
$$

The topological charge $Q$ defined by (48) is therefore proportional to the total Gaußian curvature. Finally, it is noted that the constraint $\operatorname{tr} \Phi=0$ is compatible with (53) and gives rise to classical isothermic surfaces in $\mathbb{R}^{3}$.

\section{REFERENCES}

1. K. Tenenblat, Transformations of Manifolds and Applications to Differential Equations, Pitman Monographs and Surveys in Pure and Applied Mathematics 93 (Longman, Harlow, 1998).

2. A. I. Bobenko and R. Seiler, eds., Discrete Integrable Geometry and Physics (Clarendon Press, Oxford, 1999). 
3. C. Rogers and W. K. Schief, Bäcklund and Darboux Transformations. Geometry and Modern Applications in Soliton Theory, Cambridge Texts in Applied Mathematics (Cambridge University Press, 2002).

4. R. Hirota, Discrete analogue of generalized Toda equation, J. Phys. Soc. Japan $\mathbf{5 0}$ (1981), 3785-3791.

5. B. G. Konopelchenko and W. K. Schief, Menelaus' theorem, Clifford configurations and inversive geometry of the Schwarzian KP hierarchy, J. Phys. A: Math. Gen. 35 (2002), 6125-6144.

6. A. D. King and W. K. Schief, Tetrahedra, octahedra and cubo-octahedra: integrable geometry of multi-ratios, J. Phys. A: Math. Gen. 36 (2003), 1-18.

7. W. K. Schief, Lattice geometry of the discrete Darboux, KP, BKP and CKP equations. Menelaus' and Carnot's theorems, J. Nonlinear Math. Phys. 10 (Supplement 2), (2003), 194-208.

8. M. Jimbo and T. Miwa, Solitons and infinite-dimensional Lie algebras, Publ. Res. Inst. Math. Sci. 19 (1983), 943-1001.

9. L. V. Bogdanov and B. G. Konopelchenko, Analytic-bilinear approach to integrable hierarchies. II. Multicomponent KP and 2D Toda lattice hierarchies, J. Math. Phys. 39 (1998), 4701-4728.

10. I. Ya. Dorfman and F. W. Nijhoff, On a $(2+1)$-dimensional version of the KricheverNovikov equation, Phys. Lett. A 157 (1991), 107-111.

11. L. V. Bogdanov and B. G. Konopelchenko, Möbius invariant integrable lattice equations associated with the generalized KP hierarchy, CRM Proc. Lecture Notes 25 (2000), 33-45.

12. G. Darboux, Sur une proposition relative aux equations linéaire, Compt. Rend. Acad. Sci. Paris 94 (1882), 1456-1459.

13. V. B. Matveev and M. A. Salle, Darboux Transformations and Solitons (Springer Verlag, 1991).

14. C. Wang, Surfaces in Möbius geometry, Nagoya Math. J. 125 (1992), 53-72.

15. L. P. Eisenhart, A Treatise on the Differential Geometry of Curves and Surfaces (Dover, New York, 1960).

16. B. G. Konopelchenko and W. K. Schief, A novel generalization of Clifford's classical point-circle configuration. Geometric interpretation of the quaternionic discrete Schwarzian KP equation, in preparation.

17. W. K. Clifford, A synthetic proof of Miquel's theorem, Oxford, Cambridge and Dublin Messenger of Math. 5 (1871), 124-141. $9-24$.

18. P. Ziegenbein, Konfigurationen in der Kreisgeometrie, J. Reine Angew. Math. 183(1941), 1999).

19. D. A. Brannan, M. F. Esplen and J. J. Gray, Geometry (Cambridge University Press,

20. Y. Ishimori, Multivortex solutions of a two-dimensional nonlinear wave equation, Prog. Theor. Phys. 72 (1984), 33-37.

21. B. G. Konopelchenko and B. T. Matkarimov, On the inverse scattering transform for the Ishimori equation, Phys. Lett. A 135 (1989), 183-189.

22. R. Beals and R. R. Coifman, The spectral problem for the Davey-Stewartson and Ishimori hierarchies, in A. Degasperis, A. P. Fordy and M. Lakshmanan, eds, Nonlinear Evolution Equations: Integrability and Spectral Methods (Manchester University Press, 1988), 15-23.

23. F. Pedit and U. Pinkall, Quaternionic analysis of Riemann surfaces and differential geometry, Doc. Math. J. DMV, Extra Volume ICM 1998, II (1998), 389-400. 
